\title{
La transculturalidad, elemento esencial para mejorar la atención en salud y de enfermería ${ }^{1}$
}

Institución: Universidad de Sonora, México

\author{
Blanca Escobar Castellanos ${ }^{2}$ \\ Tatiana Paravic-Klijn ${ }^{3}$
}

\section{ENSAYO}

\section{RESUMEN}

El fenómeno migratorio en distintos países es una realidad muy compleja, supone un reto para el sistema de salud, las políticas que se diseñan y los profesionales que prestan sus servicios en él. El profesional en enfermería es el responsable de la prestación de cuidados en un ámbito complejo y de adaptarse a la realidad multicultural que se vive actualmente. Este artículo tiene la finalidad de reflexionar sobre la importancia de la enfermería transcultural para fundamentar la práctica profesional basada en un cuerpo de conocimiento científico y humanizado que permita mejorar la calidad de los cuidados de enfermería culturalmente competentes con base en las necesidades del paciente/familia/comunidad y sistema de salud. A partir del enfoque transcultural se comprende las diferencias culturales existentes, y la importancia de brindar cuidados culturales integrales y de calidad considerando las creencias y valores de las personas a las que se brinda el cuidado. Al adquirir la perspectiva cultural se desarrolla la competencia cultural. Es necesario establecer propuestas de mejoras, redes de apoyo interdisciplinar y multidisciplinar nacionales e internacionales para lograr una salud integral, humanizada y culturalmente congruente tomando en cuenta las necesidades reales de la población.

Palabras clave: atención-a-la-salud, atención-de-enfermería, comunicación, cultura, enfermería-transcultural.

\footnotetext{
${ }^{1}$ Fecha de recepción: 25 de abril 2017

Fecha de aceptación: 6 de junio 2017

${ }^{2}$ Máster en Ciencias de Enfermería. Estudiante del Doctorado en Enfermería de la Universidad de Concepción, Chile. Maestra en el Departamento de Enfermería de la Universidad de Sonora, México. Correo electrónico: escobarcblanca@enfermeria.uson.mx

${ }^{3}$ Doctora en Enfermería, Profesor titular de la Facultad de Enfermería de la Universidad de Concepción, Chile. Correo electrónico: tparavic@udec.cl
} 


\title{
Transculturality, an essential element to improving health care and nursing ${ }^{1}$
}

Institution: University of Sonora, México

\author{
Blanca Escobar Castellanos ${ }^{2}$ \\ Tatiana Paravic-Klijn ${ }^{3}$
}

\section{ESSAY}

\begin{abstract}
The migratory phenomenon in various countries is a very complex reality. It poses as a challenge to the healthcare system, policies that are to be designed and the professionals providing services there. Nurses are responsible for providing care in such a complex environment and adapting to the multicultural reality that exists today. This article aims to reflect on the importance of transcultural nursing in order support the professional practice based on a body of scientific and humanized knowledge to improve the quality of culturally competent care based on the needs of the patient / family / community and health system. The transcultural approach allows us to understand cultural differences and provide comprehensive care considering the beliefs and values of the people to whom care is provided. By acquiring cultural perspective, cultural competence is developed. It is necessary to establish different approaches in order for there to be improvements, support networks of interdisciplinary and multidisciplinary national and international efforts to achieve a comprehensive, humane and culturally congruent health taking into account the real needs of the population.
\end{abstract}

Keywords: culture, communication, health-care-nursing, care-transcultural-nursing

\footnotetext{
${ }^{1}$ Date of receipt: April 25, 2017

Date of acceptance: June 6, 2017

${ }^{2}$ Master's Degree in Nursing Sciences. PhD Student in Nursing, University of Concepción, Chile. Teacher in the Nursing Department of the University of Sonora, Mexico. E-mail: escobarcblanca@enfermeria.uson.mx

${ }^{3} \mathrm{PhD}$ in Nursing, Full Professor of the Faculty of Nursisng of the University of Concepción, Chile. E mail: tparavic@udec.cl
} 


\title{
A transculturalidade, elemento essencial para melhorar a atenção na saúde e da enfermagem $^{1}$
}

\author{
Blanca Escobar Castellanos ${ }^{2}$ \\ Tatiana Paravic-Klijn ${ }^{3}$
}

Instituição: Universidade de Sonora, Chile

\section{ENSAIAR}

\section{RESUMO}

O fenômeno migratório em distintos países é uma realidade muito complexa, supõe um reto para o sistema de saúde, as políticas que se desenham e os profissionais que prestam seus serviços. O profissional em enfermagem é responsável da prestação de cuidados em um âmbito complexo e de adaptar-se a realidade multicultural que se vive atualmente. Este artigo tem a finalidade de refletir sobre a importância da enfermagem transcultural para fundamentar a prática profissional baseada em um corpo de conhecimento científico e humanizado que permita melhorar a qualidade dos cuidados de enfermagem culturalmente competentes com base nas necessidades do paciente/família/comunidade e sistema de saúde. A partir do enfoque transcultural se compreende as diferenças culturais existentes, e a importância de brindar cuidados culturais integrais e de qualidade considerando as crenças e valores das pessoas, as quais se brinda o cuidado. Ao adquirir a perspectiva cultural se desenvolve a competência cultural. É necessário estabelecer propostas de melhoras, redes de apoio interdisciplinar e multidisciplinar nacionais e internacionais para alcançar uma saúde integral, humanizada e culturalmente congruente tomando em consideração as necessidades reais da população.

Palavras-chave: atenção-à-saúde, atención-de-enfermagem, comunicação, cultura, enfermagem-transcultural

\footnotetext{
${ }^{1}$ Data de recebimento: 25 de abril de 2017

Data de aceitação: 06 junho de 2017

${ }^{2}$ Maister en Ciências da Enfermagem. Estudiante do Doutorado em Enfermagem da Universidade de Concepção, Chile. Maestra no Departamento de Enfermagem da Universidade de Sonora, México. Correo electrónico: escobarcblanca@enfermeria.uson.mx

${ }^{3}$ Doutora en Enfermería, Professora Titular da Faculdade de Enfermagem da Universidade de Concepção, Chile. Correo electrónico: tparavic@udec.cl
} 


\section{Revista Electrónica Enfermeria Actual en costa Rica}

\section{INTRODUCCIÓN}

En la actualidad, la atención a la salud debe otorgarse a una sociedad cada vez más diversificada y globalizada ${ }^{1}$, la cual recibe la influencia de múltiples factores que afectan a las personas, a lo que se suma las diferencias culturales de quienes la integran y la condicionan, no solo en las actuaciones de tipo social, económicas o culturales, sino en el ámbito de los problemas o necesidades de salud.

El fenómeno migratorio evoca una realidad muy compleja para distintos países, "lo cual supone un reto para el sistema de salud, para las políticas que se diseñan y para todos los profesionales que prestan sus servicios en él, principalmente para el personal de enfermería", responsable de la prestación de cuidados en un ámbito complejo y de adaptarse a la realidad multicultural, debido a la influencia que la cultura tiene en el proceso saludenfermedad de los individuos a los que atiende y dirige los cuidados tanto en el ámbito hospitalario como comunitario $^{2}$.

Algunos países se han preocupado por las disparidades de la atención de la salud originadas por las desigualdades culturales en materia de acceso al cuidado de la salud, prestación de asistencia sanitaria de calidad y resultados de salud. Las desigualdades en salud principalmente en "la edad, género, raza, etnia, educación, ingresos, discapacidad, residencia y orientación sexual de la población, son una preocupación alarmante, por lo que organizaciones profesionales, instituciones educativas de nivel superior y algunos organismos involucrados en la salud intentan abordar esta situación", centran su objetivo en lograr "la competencia cultural individual y organizacional" para mejorar la atención a la salud y poder abatir las disparidades ocasionadas por la diversidad multicultural actual",3.

Para brindar la atención dentro del sistema de salud, se debe considerar los aspectos de cuidado comunicación y cultura, estrechamente relacionados, debido a que la comunicación es parte escencial de la conducta humana. Al respecto, González (1989) citado por Barbón hace referencia que la comunicación puede entenderse como "intercambio, interrelación, como diálogo, como vida de sociedad, todo ello relacionado indisolublemente con las necesidades sociales del hombre y no puede existir sin el lenguaje" , mientras que la cultura permite conocer los modos de vida y costumbres, conocimientos y grado de desarrollo de las personas o grupo social de una cultura particular, son aprendidos, practicados y generalmente transmitidos de generación en generación a través de la comunicación, mas se convierten en una barrera cuando la comunicación u otros factores intervienen en ella. Dado lo anterior, existe la necesidad de enfrentar las barreras culturales, como el lenguaje (entendido como el sistema de comunicación que permite formular conceptos entre las personas de las diversas culturas) para facilitar el cuidado enfermero de calidad ${ }^{5}$.

Para integrar tales conceptos (comunicación, cultura y cuidado) en los últimos años se ha incorporado el término "transcultural", entendido como "un fenómeno que afecta a varias culturas o a sus relaciones", y que ocurre cuando un grupo social recibe y adopta las culturas que provienen de otros grupos, de modo que la 


\section{Revista Electrónica Enfermeria Actual en costa Rica}

comunidad acaba sustituyendo en mayor o menor medida sus propias prácticas culturales y profesionales, así como el idioma, forma de vestir, entre otras características.

A partir de la cultura y el cuidado, surge la enfermería transcultural, rama de la enfermería con influencia de la Antropología, cuyo foco principal es el estudio y análisis comparativo de diferentes culturas o subculturas ${ }^{4}$.

El personal de enfermería con formación transcultural debe tomar en cuenta las creencias culturales, las conductas del cuidado y los valores de los individuos, familias y grupos para proporcionar cuidados enfermeros efectivos, satisfactorios y coherentes, con el fin de desarrollar un cuerpo de conocimiento humanístico y científico organizado para proveer una práctica de enfermería, culturalmente específica y universal ${ }^{6}$. Para lograrlo es importante desarrollar la investigación en un marco teórico que considere las diversidades culturales como eje central para otorgar cuidados de enfermería culturalmente competentes, cuyo resultado será mejorar la calidad de atención a la población que vive o se desarrolla con una cultura diferente a su contexto cultural, lo cual mejorará el quehacer profesional, partiendo de la percepción de la cultura propia y de las personas a las cuales se atiende. El resultado será un trato humanizado, mejor calidad de la atención y comunicación que evidencien la adquisición de la competencia cultural en los servicios de salud.

El presente trabajo tiene la finalidad de reflexionar sobre la importancia de la enfermería transcultural para fundamentar la práctica profesional basada en un cuerpo de conocimiento científico y humanizado, cuyo fin sea mejorar la calidad de los cuidados de enfermería culturalmente competentes con base en las necesidades del paciente/familia/comunidad y sistema de salud.

\section{DESARROLLO}

\section{Transculturalidad para la atención de la salud}

Comprender términos como cultura, multiculturalidad, interculturalidad y el propio concepto de transculturalidad, es fundamental para aclarar el enfoque y la trascendencia que tienen en el ámbito de la salud y sobre todo su relevancia para la enfermería. Actualmente, los fenómenos migratorios generados en la mayoría de los países han permitido modificar el concepto cultura el cual ha sufrido diferentes transformaciones.

En cuanto a la cultura como tal, surge en Alemania a finales del siglo XVIII; proviene del latín colere que significa "cultivar" y se refiere al esfuerzo humano para cultivarse, progresar hacia los valores de una cultura por excelencia. Desde el punto de vista antropológico, enfatiza el saber colectivo y característico de todos los grupos sociales. En torno al concepto, Tylor mencionó que la cultura o civilidad entendida en su más amplio sentido etnográfico es aquel conjunto que comprende el conocimiento, las creencias, el arte, la moral, el derecho, las costumbres y todas las capacidades y hábitos adquiridos por parte del hombre como miembro de una sociedad.

La cultura permite delimitar el "qué cosa hacer", "cómo hacerla" y "por qué se hace", de forma consciente o inconsciente condicionada por las normas culturales que influirán en el proceso de aprendizaje de los grupos sociales ${ }^{7}$. 


\section{Revista Electrónica Enfermeria Actual en costa Rica}

Según Lara y Cárdenas, cada cultura "establece su propia forma de comprender, adecuar y clasificar su mundo de acuerdo con su ideología; esta define representaciones y creencias con las que se concretan formas de acción a nivel político, religioso, filosófico y en salud". A partir de esta ideología se determina las concepciones sobre salud, enfermedad, dolor y muerte, las cuales influyen en la forma de prevenir y promover la salud, curar las enfermedades y conservar la vida ${ }^{8}$.

La definición de cultura está relacionada con la interculturalidad, puesto que existen diferentes culturas, grupos sociales diferentes, es decir, existe la cultura específica del género humano y que es común a todos los hombres, pero está aquella con características propias y variables de cada grupo humano.

Todas las culturas presentan un patrón universal similar desde el punto de vista antropológico: al respecto, Harris considera que la superestructura toma en cuenta la creatividad, ideología y valores (ideología y valores religiosos, éticos, morales y estéticos, actividades lúdicas, artísticas, creativas y expresivas) y aspectos intelectuales y mentales (organización psicológica, cognitiva y afectiva, organización y sistema educativo formal y no formal) $)_{7}$. La estructura aborda la economía política (relaciones externas, sistema de distribución y acceso al poder, poder territorial, militar y judicial) y la economía doméstica (sistema familiar, parentesco, clan, papel de la mujer, relaciones intergeneracionales, sistemas de consumo, ahorro, distribución y reparto). Por último, la infraestructura considera el modo de reproducción (regulación del crecimiento demográfico, técnicas y ritos de crianza y educación intrafamiliar) y el modo de producción (requisitos mínimos de subsistencia como artesanía, herramientas, vivienda, alimentación, cocina, vestuario, salud, recolección, agricultura, caza, pesca, entre otros).

De igual forma, Konthak propone tres niveles de cultura: primero la cultura nacional que permite conocer las experiencias, creencias, patrones de comportamiento y valores compartidos por ciudadanos del mismo país; segundo, la internacional, que se extienden más allá de los límites nacionales, donde dos culturas pueden compartir experiencias y medios de adaptación a través del préstamo o la difusión de la cultura de forma directa o indirecta; y la tercera se refiere a la subcultura, la cual incluye los patrones y tradiciones basadas en símbolos diferentes asociados a subgrupos que conviven en la misma sociedad compleja ${ }^{7}$.

La cultura tiene un carácter estático y dinámico, de este último surge el fenómeno de la multiculturalidad que forma parte del núcleo de nuestro contexto actual. Hablar de multiculturalidad (prefijo "multi" muchos) hace referencia a la existencia de varias culturas diferentes en el mismo lugar, sin establecer relación entre ellas. Algunos autores coinciden que la multiculturalidad es "única y exclusivamente la yuxtaposición de las distintas culturas existentes en un mismo espacio físico, pero sin que implique que haya un enriquecimiento, es decir, sin que haya intercambio entre ellas", por tanto, la sociedad y sus individuos serán multiculturales si mantienen un estado de indiferencia o de tolerancia por las diversas culturas ${ }^{7}$ mas, cuando se hace referencia a las prácticas multiculturales para preservar la identidad étnica de los pueblos, al considerar a los "otros" desde una posición de superioridad estructural, para marginarlos del grupo, se establece una diferencia de desigualdad.

Cuando se recurre al término interculturalidad (prefijo "inter" entre), involucra la relación, intercambio y comunicación comprensiva entre las diferentes culturas que conviven en un mismo espacio, originando el enriquecimiento mutuo, el reconocimiento y la valoración (intrínseca y extrínseca) entre las distintas culturas en un marco de igualdad. La interculturalidad permite crear la diversidad cultural, intenta comprender las diferencias 


\section{Revista Electrónica Enfermeria Actual en costa Rica}

culturales, permite la participación de los ciudadanos para derribar las barreras culturales. Se consideran interculturales si se establecen relaciones interactivas entre las diferentes realidades presentes.

El fenómeno de la interculturalidad se considera una filosofía (ofrece un marco teórico que permite ver e interpretar la realidad), un proceso (aporta un modelo racional para organizar ideas y esfuerzos) y un programa (plantea una forma sistemática para organizar actividades dirigidas a la creación de un medio intercultural $)_{7}$.

Por su parte, Herskovits (1995), citado por Tarres, menciona que la transculturalidad son "aquellos fenómenos que resultan cuando los grupos de individuos, que tienen culturas diferentes, toman contacto continuo de primera mano, con los consiguientes cambios en los patrones de la cultura original de uno de los grupos o de ambos" ". El término se analiza como un proceso que permite el acercamiento entre las diferentes culturas (incide en los sistemas de creencias y valores), busca establecer vínculos más allá de la misma cultura en cuestión, casi creando hechos culturales nuevos que nacen del sincretismo (sistema que permite la conciliación de diferentes doctrinas): si lo anterior se facilita, la convivencia de la cultura a través de los medios de comunicación e información, puede favorecer que la sociedad manifieste respeto, acuerdos y promoción de valores universales por encima de las particularidades de raza, etnias y creencias religiosas, de modo que se dé la fraternidad universal o el principio hacia una "transculturalidad"?

En el contexto de la diversidad cultural se perciben dos conceptos importantes para entender la cultura, pero ambos son opuestos, a) el relativismo cultural que "consiste en la capacidad de comprender las creencias y costumbres de otros pueblos o sociedades desde el contexto de su propia cultura, partiendo de que todas las culturas son iguales y ninguna es superior a otra" y b) el etnocentrismo, "tendencia a juzgar las creencias y costumbres de otras sociedades desde la propia cultura". En relación con ambos aspectos de la diversidad cultural, el personal de enfermería no está preparado para enfrentar a la población con necesidades culturales diferentes en salud, lo que implica que se sientan inseguras al brindar el cuidado y se convierte en un obstáculo para establecer una relación terapéutica, motivo por el que es necesario desarrollar el relativismo cultural al comprender a la persona desde el contexto de su propia cultura, para brindar cuidados culturalmente competentes.

En cuanto al etnocentrismo, afecta la calidad de la atención que se otorga a las personas de culturas diferentes, lo que "supone un obstáculo para la mejora de la salud de la persona"10, al no considerar su cultura en el momento de establecer un diagnóstico o tratamiento y/o cualquier modo de comunicación durante la práctica asistencial $^{11}$.

A pesar de los importantes cambios, el modelo de salud aun no responde a las demandas de la nueva realidad multicultural, por lo que es indispensable que se establezcan modelos que consideren la diversidad cultural producto de la globalización, la tecnología y la migración, en materia de salud y enfermedad ${ }^{12}$. Las diferencias culturales entre los profesionales de la salud y los pacientes pueden convertirse en impedimentos que afectan los resultados del cuidado de la salud ${ }^{13}$. Santágata y Terrasa, mencionan que es necesario que los sistemas de salud enfrenten esta situación para aumentar la calidad de la asistencia en salud en relación con la accesibilidad, aceptación, eficiencia y satisfacción de las personas que pertenecen a una cultura diferente a la de quienes brindan los servicios ${ }^{14}$.

Según Fuller, los profesionales de enfermería al desarrollar su trabajo deben tener en cuenta que confluyen al menos con tres culturas: la del paciente, su propia cultura y la de la organización donde trabajan ${ }^{15}$. 


\section{Revista Electrónica Enfermería Actual en costa Rica}

\section{La enfermería transcultural}

En el ámbito de la enfermería profesional, como concepto surge la enfermería transcultural en el año de 1950, la cual intentó unir la práctica de la enfermería con el conocimiento antropológico, para brindar un mejor cuidado a los pacientes cada día más heterogéneos ${ }^{9}$. El desarrollo de esta área surge ante la diversidad cultural creciente que caracteriza a las poblaciones dentro y entre los países nacional y mundialmente. La enfermería transcultural permite descubrir los puntos de vista personales o culturales, referentes a los cuidados para entenderlos, aplicarlos y aprovechar estos conocimientos como base de las prácticas asistenciales. ${ }^{16}$ Leininger menciona que es "un área formal de conocimientos y prácticas humanísticas y científicas centrada en fenómenos y competencias del cuidado de enfermería enfocada en el cuidado cultural holístico para ayudar a individuos y grupos a mantener o recuperar su salud/bienestar, para afrontar discapacidades, la muerte $u$ otras condiciones humanas, de forma culturalmente coherente y beneficiosa"17,18. La enfermería se enfrenta al multiculturalismo lo cual supone un reto y una oportunidad para la disciplina, al transformar la práctica profesional en cuidados culturalmente competentes, al convertirse en agente de cambio en los sistemas de salud.

Cuando se aborda la diversidad cultural para brindar el cuidado desde la perspectiva de enfermería, se emplea múltiples términos como enfermería transcultural, intercultural e internacional, los cuales son utilizados de forma indistinta, por lo que causan confusión; sin embargo cada uno de ellos tienen una perspectiva propia. En relación con lo anterior, para Leininger, la enfermería transcultural permite a los profesionales de enfermería prepararse y comprometerse a desarrollar conocimientos y prácticas en la transculturalidad, se enfocan a varias culturas con una base teórica y práctica de enfermería comparativa, mientras que la intercultural alude a los profesionales de enfermería que aplican conceptos antropológicos y la enfermería internacional se centra en profesionales capaces de trabajar entre dos culturas ${ }^{17}$.

Madeleine M. Leininger fue la fundadora y líder en la teoría de los cuidados culturales a las personas (teoría de la diversidad y de la universalidad), desarrolló este enfoque desde la antropología cultural, social y psicológica y de la enfermería. El desarrollo de sus investigaciones ha permitido entender las diferencias culturales existentes en los cuidados humanos, la salud y la enfermedad ${ }^{17}$. La etnoenfermería permitió el progreso de la enfermería transcultural al centrar el estudio de las creencias, valores y prácticas empleados en la asistencia de enfermería de acuerdo con los conocimientos cognitivos o subjetivos ${ }^{19}$ sobre una cultura en particular, expresados mediante el lenguaje, experiencias y sistema de valores aplicados a fenómenos de enfermería como la asistencia, la salud y los factores ambientales ${ }^{20}$. El método de investigación cualitativa en etnoenfermería permitió al profesional de enfermería captar el punto de vista interno de las culturas al analizar los complejos cuidados y los fenómenos culturales.

La relevancia del desarrollo de la enfermería transcultural ha permitido implementar el cuidado de enfermería profesional con énfasis en la transculturalidad, considerada "un área principal de la enfermería que se centra en el estudio comparativo y en el análisis de las diferentes culturas y subculturas del mundo con respecto a los valores sobre los cuidados, la expresión y las creencias de la salud y la enfermedad y el modelo de conducta". La finalidad es "descubrir las particularidades y las universalidades de los cuidados de los humanos según la visión del mundo, la estructura social y otras dimensiones y después descubrir formas de proporcionar cuidados 


\section{Revista Electrónica Enfermeria Actual en costa Rica}

culturalmente congruentes a personas de culturas diferentes o similares para mantener o recuperar su bienestar, salud o afrontamiento con la muerte de una manera culturalmente adecuada"17.

El modelo teórico para la enfermería transcultural de Leininger, "el modelo del sol naciente", se desarrolló en 1970 y describe a los seres humanos como entes que no se pueden separar de su procedencia cultural y de la estructura social, de su concepción del mundo, de su trayectoria de vida y del contexto de su entorno. Señala que los cuidados y la atención están influenciados por el lenguaje, la etnohistoria y el entorno como: los valores culturales, creencia y estilos de vida y factores de tipo tecnológico, religiosos y filosóficos, familia y factores sociales, políticos, legales, económicos y educacionales. Además, permite determinar las decisiones y acciones de los cuidados por parte de enfermería, para desarrollar cuidados culturalmente coherentes encaminados a la preservación y mantenimiento de los cuidados culturales, adaptación y negociación de los cuidados culturales y reorientación y reestructuración de los cuidados culturales ${ }^{18,19}$.

Posterior al modelo de Leininger, surgen propuestas teóricas que permiten sustentar la enfermería transcultural, siendo similares en sus principios: estos marcos teóricos engloban las estrategias que los individuos utilizan para mantener, proteger y recuperar su salud física, mental y espiritual considerando los cuidados transculturales $^{11}$, entre los que se encuentra Rachel E. Spector con el de Herencia cultural y Tradiciones de salud $^{21}$; Larry Purnell, con el de la Competencia cultural ${ }^{22}$; Josepha Camphina-Bacote, quien implementó el Proceso de Competencia cultural en la prestación de los servicios de cuidados de salud ${ }^{23}$ y el modelo de Juliene Lipson $^{24}$.

Cuando enfermería emplea un modelo teórico con enfoque transcultural para sustentar su práctica profesional se esperaría que los beneficios para la población que atiende sea brindar cuidados culturales holísticos y congruentes con sus necesidades, debido a que la diversidad cultural promueve que la sociedad sea cada vez más compleja, diversa y con múltiples matices culturales. Al hacer referencia a los cuidados culturales, Leininger menciona que son "actos de prestación de cuidados de ayuda, apoyo, facilitación o capacitación sintetizados y culturalmente constituidos hacia uno mismo o hacia los demás, centrados en necesidades evidentes o anticipadas para la salud o el bienestar del cliente o para afrontar discapacidades, la muerte u otras condiciones humanas"16.

Para Spector los cuidados de enfermería para ser considerados "cuidados culturales deben ser culturalmente sensibles y competentes, un cuidado culturalmente sensible implica que se poseen conocimientos básicos y actitudes constructivas hacia las tradiciones de salud observadas en los diversos grupos culturales en el lugar en el que se está trabajando"21,25. Desde la óptica de la cultura, analizar el cuidado humano implica comprender que es inseparable y propio del ser humano, el cual es diverso, plural, universal y complejo, por lo tanto, cuidar está influenciado por la cultura de quien proporciona y de quien recibe el cuidado ${ }^{26}$.

La importancia al desarrollar los cuidados culturales permite considerar la vida humana desde la perspectiva holística, o sea a la persona con sus múltiples referencias culturales, significados de vida y salud, valores o modos de vivir, su estructura social, las formas de comunicación y contexto en general, para devolver el bienestar al paciente, al conducir las prácticas del cuidado de enfermería desde la perspectiva transcultural: cuando el personal de enfermería ha sido capaz de involucrar la integración de conciencia cultural, conocimientos culturales, habilidades culturales, encuentros y deseos culturales se dice que logró "la competencia cultural"27. 


\section{Revista Electrónica Enfermeria Actual en costa Rica}

Esta competencia cultural no es exclusiva para el personal de enfermería sino para todos aquellos que trabajan en situaciones de interculturalidad, motivo por el que la oficina de las Minorías en Salud de Estados Unidos de América en 2014 definió la competencia cultural y lingüística como "un conjunto de comportamientos congruentes, actitudes y políticas que al unirse en un sistema, agencia o entre profesionales permite un trabajo eficaz en situaciones interculturales" ${ }^{\prime 3}$, lo cual implica que el personal de enfermería debe desarrollar una perspectiva cultural que le permite reconocer "la propia cultura y la de otras personas a las que atiende" con el fin de brindar cuidados culturalmente aceptables, basados en los aspectos biofísicos, culturales, sociales y ambientales ${ }^{19,28}$.

\section{Investigación en enfermería transcultural}

La investigación en enfermería transcultural se considera importante para el avance del conocimiento de la disciplina, aun cuando existe la necesidad de contar con una perspectiva global; sin embargo, la literatura en investigación de enfermería en esta área ofrece poca información ${ }^{29}$. Es necesario contar con estudios comparativos plurinacionales e internacionales en grupos minoritario o específicos con culturas similares a partir de los que se proponga cambios a nivel de las políticas en salud, para establecer reformas a nivel de los sistemas y organizaciones $^{22,28}$.

$\mathrm{Al}$ interior de los países, es prioritario obtener información sobre las variaciones culturales de la población que se atiende, evidenciar las necesidades de mejoras, los cambios generados en los procesos de la atención o sistemas de organización y cuáles han sido los sus resultados; no obstante, es ineludible establecer redes de investigación de enfermería transcultural internacional y nacional, así como inter y multidisciplinar para crear una base de datos global que sustente las necesidades de las poblaciones y las propuestas para abordar las problemáticas identificadas en salud por grupos minoritarios o vulnerables.

Otro aspecto necesario consiste en identificar las barreras en los sistemas de comunicación verbal (lenguaje/idioma) y no verbal de las personas en su diversidad cultural con el equipo de salud, la cual permite integrar la conducta y favorece los procesos cognitivos y afectivos ${ }^{13,30}$; de igual modo, es fundamental identificar algunas de las reacciones psicológicas o físicas observadas en la población de migrantes o poblaciones con características diferentes a la cultura "predominante" de la región, ya que pueden manifestar reacciones defensivas dentro del proceso de adaptación a una nueva cultura, debido a que los cambios culturales provocan una etapa de transición al enfrentarse a diferentes modos de vida o de cultura que cambia de una sociedad a otra que generan en el individuo o familia un "ajuste personal y nuevo aprendizaje social al enfrentarse a nuevas culturas o entornos" $"$.

En el ámbito asistencial, se debe evidenciar la aplicabilidad de los modelos teóricos de la enfermería transcultural tomando en cuenta los fenómenos de estudios específicos de la enfermería y los resultados de su abordaje para mejorar la práctica del cuidado, por ciclo de vida, grupo étnico, grupos etarios, género, minorías de raza o creencias religiosas, lo cual sustentará las intervenciones para la prestación de los servicios a las personas o grupos. También es importante contar con instrumentos validados en español para identificar las necesidades culturales de la población y las competencias culturales del personal de salud, así como establecer un sistema de diálogo o "negociación" entre el profesional de enfermería y el paciente/familia para determinar -en conjunto- la forma de implementar los cuidados culturales, respetando las normas de la institución de salud y los 


\section{Revista Electrónica Enfermeria Actual en costa Rica}

deseos/costumbres de respeto de la cultura o religión del paciente ${ }^{12}$; es decir, enfermería no debe separar el patrón cultural, de salud y del cuidado al planificar las intervenciones ${ }^{31}$.

Como componente prioritario en la preparación de los profesionales en enfermería ${ }^{32}$, se debe fomentar el desarrollo de la competencia cultural para abordar la investigación transcultural, logro que se obtiene al incorporar tópicos de mayor impacto de tipo social o en salud derivados de la diversidad cultural en los planes de estudio de formación básica o de posgrado. Entre algunos de esos temas, se encuentra los retos de la salud mundial, los sistemas de entrega de atención a la salud, las teorías y modelos transculturales, la comunicación intercultural, las creencias de salud y enfermedad de base cultural y prácticas, bases culturales en las modalidades de curación y atención, evaluación de la salud cultural, roles profesionales y atributos de enfermería transcultural ${ }^{33,34}$.

A partir de lo mencionado, es imprescindible que el profesional de enfermería y los futuros graduados cuenten con bases teóricas en enfermería transcultural con enfoque cualitativo y etnoenfermería ya que, como sugiere Leininger, contribuyen a desarrollar el estudio y clasificación sistemática de las creencias, valores y prácticas que se implementan en el cuidado de enfermería desde etapas tempranas de formación, al adquirir conocimientos sobre determinada cultura a través de las manifestaciones generales o específicas que demuestra la persona/familia/comunidad, expresadas por el lenguaje, experiencias, convicciones y sistemas de valores, sobre fenómenos de enfermería reales o potenciales en las áreas asistenciales, la salud-enfermedad y factores ambientales o sociales ${ }^{29}$.

Por último, se destaca la necesidad de incorporar los aspectos éticos en el cuidado de la enfermería transcultural, aplicando siempre los principios de respeto por las personas en su cultura, ideologías, creencia y valores (reconocimiento a la autonomía), la beneficencia (maximizar los beneficios posibles), la justicia (manejo equitativo e igualitario de los sujetos) y la no maleficencia (no hacer daño), solicitando siempre el consentimiento informado ${ }^{35}$, sin olvidar que la persona como un ser social con derechos inalienables.

\section{CONCLUSIONES}

La globalización y la migración de poblaciones son fenómenos complejos en diversos países que ocasionan demandas en diversos ámbitos de la sociedad, principalmente en la atención a la salud de la población. El sistema de salud debe responder a esa necesidad que surge ante la demanda social; sin embargo, tal acción supone un reto al tratar de abatir las desigualdades generadas por condiciones de tipo cultural, edad, sexo, genero, etnia, residencia, entre otros aspectos que son propios de grupos o colectivos que solicitan acceso al cuidado de la salud y a la prestación de la asistencia sanitaria, pero que presentan una diversidad cultural que genera barreras para otorgar servicios de salud acordes a sus necesidades. Ante la diversidad cultural que predomina, enfermería no puede quedarse al margen, debe considerar la cultura, la comunicación y el cuidado dentro del contexto sanitario como elementos importantes para desarrollar la enfermería transcultural.

El personal de enfermería con formación transcultural debe considerar a la persona en su totalidad para brindar cuidados holísticos, humanizados, culturalmente competentes y congruentes con base en las creencias culturales, las conductas del cuidado y los valores de los individuos, familias y grupos a los cuales brinda el cuidado. 


\section{Revista Electrónica Enfermeria Actual en costa Rica}

La enfermería transcultural es una vía para descubrir los puntos de vista personales o culturales, referentes a los cuidados para entenderlos, aplicarlos y aprovecharlos como base en modelos teóricos de la enfermería transcultural: al integrar tal visión, se logrará la competencia de los cuidados culturales.

Por último, se debe dar importancia al desarrollo de la investigación, ya que permite adquirir y desarrollar un cuerpo de conocimientos científicos y humanísticos en la práctica de enfermería culturalmente específica y universal, partiendo del propio conocimiento que se tenga de la cultura y del que se adquiere de la cultura de los "otros", a quienes se brinda el cuidado, por tanto, el profesional debe desarrollar habilidades y actitudes para su conocimiento y aplicación, cuyo beneficio será evidenciar los resultados de las investigaciones en grupos específicos para fundamentar las intervenciones de enfermería o programas que se desarrollan para generar cambios en el personal; del mismo modo, la organización debe adoptar políticas o estrategias para mejorar la calidad de atención que se brinda y abatir las desigualdades producto de las barreras ocasionadas por la diversidad cultural.

\section{REFERENCIAS BIBLIOGRÁFICAS}

1. Barco DV, Cruz AS, Rodríguez BZ, Herrera CD. Gestión del cuidado desde una perspectiva transcultural. Rev. Cubana Enfermer.[Internet] 2011;27(1):57-65. Disponible en http://scielo.sld.cu/scielo.php?script=sci_arttext\&pid=S086403192011000100007

2. Fernández MM. Estudio de los cuidados a pacientes extranjeros hospitalizados desde la perspectiva de la enfermería transcultural. [Tesis doctoral]. Consejo de enfermería de la comunidad valenciana. [Internet]. 2006. Disponible en http://www.bibliotecadigitalcecova.com/contenido/revistas/cat6/pdf/libro 50.pdf

3. Bauer k, Bai Y. Innovative educational activities using a model to improve cultural. Procedia Social and Behavioral Sciences. [Internet] 2015; 174:705-710. Disponible en http://www.sciencedirect.com/science/article/pii/S1877042815006564

4. Barbón PO. Cultura y enfermería transcultural, herramientas para la competencia comunicativa de un enfermero internacionalista. Rev de Actualizaciones en Enfermería. [Internet] 2011;14(3). Disponible en https://encolombia.com/medicina/revistas-medicas/enfermeria/ve-143/culturayenfermeria/

5. Islas SP, Zesati PG, Moye EM. Una propuesta educativa en enfermería transcultural: El caso de la cultura menonita. Rev Enfermería Universitaria ENEO-UNAM. [Internet] 2011;8(3):1-7. Disponible en http://www.revistas.unam.mx/index.php/reu/article/view/31374

6. Fernández GV. Perspectiva de la enfermería transcultural en el contexto mexicano. Rev Enferm IMSS. [Internet] 2006;14 (1): 51-55. Disponible en http://www.medigraphic.com/pdfs/enfermeriaimss/eim-2006/eim061j.pdf 


\section{Revista Electrónica Enfermeria Actual en costa Rica}

7. Hidalgo HV. Cultura, Multiculturalidad, Interculturalidad y transculturalidad: Evolución de un término. [Internet] 2005. 73-83. Disponible en http://pedagogia.fcep.urv.cat/revistaut/revistes/juny05/article04.pdf

8. Laza VC, Cárdenas MF. Abordaje interdisciplinario de la salud y la enfermedad: Aportes a la enfermería. Index Enferm. [Internet] 2009;18(1):37-41. Disponible en http://scielo.isciii.es/scielo.php?pid=S1132-

$\underline{12962009000100008 \& \text { script }=\text { sci arttext }}$

9. Tarrés CS. El cuidado del "otro" diversidad cultural y enfermería transcultural. Gazeta de Antropología. [Internet] 2001;17(15):1-6. Disponible en: http://www.gazeta-antropologia.es/?p=3267

10. Pértega AE, López RM, Hospital MA, Baonza GS. Efectividad de un programa formativo en enfermería transcultural sobre aspectos de la salud mental en hijos de inmigrantes entre 12 y 17 años diagnosticados de síndrome de estrés por traslado. Nure investigación. [Internet] 2008;44. Disponible en http://www.nureinvestigacion.es/OJS/index.php/nure/article/view/473

11. Fernández O A, Mgueraman JR, Rojas MM. Enfermería sin fronteras. Enfermería y el paciente musulmán. Enfermería Docente. [Internet] 2011;94:16-21. Disponible en http://www.juntadeandalucia.es/servicioandaluzdesalud/huvvsites/default/files/revistas/ED-094-05.pdf

12. Soriano AE, González JA, Cala VC. Retos actuales de educación y salud transcultural. Tomo1, Vol. 1. 2014.

13. González JL, Noreña PM. Comunicación intercultural como medio para favorecer el cuidado culturalmente aceptable. Enfermería Universitaria. [Internet] 2000;8(1):55-60. Disponible en http://www.revistas.unam.mx/index.php/reu/article/view/25472.

14. Santágata M, Terrasa S. Asistencia médica basada en la diversidad cultural. Rev Evidencia. [Internet] 2002;5(3):79-81. Disponible en http://www.foroaps.org/files/transcultural.pdf

15. Plaza del Pino F, Soriano AE. Formación de los profesionales de enfermería: Cuidar en la sociedad multicultural del siglo XXI. Index Enferm. [Internet] 2009;8(3). Disponible en http://scielo.isciii.es/scielo.php?script=sci_arttext\&pid=S1132-12962009000300011

16. Llanes BC. La transculturación en el cuidado de enfermería gerontológica comunitaria. Rev. Cubana Enfermer. [Internet] 2012;28(3):195-208. Disponible en http://scielo.sld.cu/scielo.php?script=sci arttext\&pid=S0864$\underline{03192012000300004}$

17. Raile AM. Teoría de la diversidad y de la universalidad de los cuidados culturales. Modelos y teorías en enfermería. 8 a ed. España: Elsevier; 2015. p. 405-429 


\section{Revista Electrónica Enfermeria Actual en costa Rica}

18. Muñoz de Rodríguez L, Vásquez M. Mirando el cuidado cultural desde la óptica de Leininger. Colomb Med. [Internet] 2007;38(2):98-104. Disponible en

http://colombiamedica.univalle.edu.co/index.php/comedica/rt/printerFriendly/542/962

19. Fornons FD. Madeleine Leininger: Claroscuro trascultural. Index Enferm. [Internet] 2010;19(2-3) Disponible en http://scielo.isciii.es/scielo.php?script=sci_arttext\&pid=S1132-12962010000200022

20. Cárcamo OM. Una propuesta de potenciación de los programas de salud intercultural, desde la perspectiva de la enfermería transcultural. [Tesis Pregrado]. Universidad Austral de Chile [Internet]. 2009. Disponible en http://cybertesis.uach.cl/tesis/uach/2009/fmc265p/doc/fmc265p.pdf

21. Spector RE. Enfermería de cuidados culturales. Cultura de los cuidados. [Internet]. 2002;6(11):5-10. Disponible en https://rua.ua.es/dspace/bitstream/10045/4857/1/CC_11_01.pdf

22. Purnell LD. El modelo de competencia cultural de Purnell: Descripción y uso en la práctica, educación, administración e investigación. Cultura de los Cuidados [Internet]. 1999;3(6):91-97. Disponible en:

http://rua.ua.es/dspace/handle/10045/5157.

23. Romero MN. Investigación, cuidados enfermeros y diversidad cultural. Index Enferm. [Internet]. 2009:18(2).

Disponible en http://scielo.isciii.es/scielo.php?script=sci_arttext\&pid=S1132-12962009000200007

24. Lipson JG. Cultura y Cuidados de Enfermería. Index Enferm. [Internet]. 2000;28-29. Disponible en http://www.indexf.com/index-enfermeria/28-29revista/28-29_articulo_19-25.php?textclock1=

25. Bonill de las Nieves Candela, Celdrán Mañas Miriam. El cuidado y la cultura: Génesis, lazos y referentes teóricos en enfermería. Index Enferm [Internet]. 2012 ; 21( 3 ): 160-164. Disponible en:

http://scielo.isciii.es/scielo.php?script=sci_arttext\&pid=S1132-12962012000200011\&lng=es.

http://dx.doi.org/10.4321/S1132-12962012000200011.

26. Reina R, Ferrer RX, Toro Y, Cárdenas MH. Abordaje del cuidado neonatal: un enfoque transcultural. Enfermería Global. [Internet]. 2010; 9(3):1-5. Disponible en http://revistas.um.es/eglobal/article/view/110961

27. Marrero GC. Competencia cultural. Enfoque del modelo de Purnell y Campinha-Bacote en la práctica de los profesionales sanitarios. Ene Revista de Enfermería. [Internet]. 2013;7(2):1-9. Disponible en: http://eneenfermeria.org/ojs/index.php/ENE/article/view/278

28. Leno GD. Buscando un modelo de cuidados de enfermería para un entorno multicultural. Gazeta de Antropología. [Internet]. 2006; 22:1-14. Disponible en http://www.ugr.es/ pwlac/G22_32Daniel_Leno_Gonzalez.html 


\section{Revista Electrónica Enfermeria Actual en costa Rica}

29. Suhonen R, Saarikoski M, Leino-Kilpi H. Cross-cultural nursing research. International Journal of NursingStudies. [Internet]. 2009;46:593-602. Disponible en http://www.journalofnursingstudies.com/article/S0020-7489(08)002459/pdf

30. Friedemann M, Pagan-Coss H, Mayorga C. The workings of a multicultural research team. J TranscultNurs. [Internet]. 2008;19(3):1-11. Disponible en: https://www.ncbi.nlm.nih.gov/pmc/articles/PMC3809833/

31. Castillo MJ. Cuidado cultural de enfermería. Necesidad y relevancia. Revista Habanera de Ciencias Médicas. [Internet]. 2008;3(7):2-7. Disponible en http://scielo.sld.cu/scielo.php?script=sci arttext\&pid=S1729-519X2008000300003

32. Chiang-Hanisko L, You-Qing P. Efficacies of different methods of teaching transcultural nursing practice in China. Chinese Nursing Research. [Internet]. 2014;1:17-24. Disponible en: http://www.sciencedirect.com/science/article/pii/S2095771815000031

33. Prosen M. Introducing transcultural nursing education: Implementation of transcultural nursing in the postgraduate nursing curriculum. Procedia Social and Behavioral Sciences. [Internet]. 2015;174:149-155. Disponible en: http://www.sciencedirect.com/science/article/pii/S1877042815006916

34. Sheets CS, Sheerin F, Bancel S, Rodrigues GJ. Curriculum meeting points: A transcultural and transformative initiative in nursing education. Nurse Education in Practice. [Internet]. 2012;12(6):304-309. Disponible en: http://www.sciencedirect.com/science/article/pii/S1471595312000637?via\%3Dihub

35. Aristizábal FL. El porqué de la ética en la investigación científica. Investig andina. [Internet]. 2012;14(24):369-371. Disponible en http://www.scielo.org.co/scielo.php?script=sci arttext\&pid=S0124-81462012000100001 